\title{
A new discrete dipole kernel for quantitative susceptibility mapping
}

Carlos Milovic ${ }^{\mathrm{a}, \mathrm{b}}$ (corresponding author, cmilovic@uc.cl), Julio Acosta-Cabronero ${ }^{\mathrm{c,d}}$ (jac@cantab.net), José Miguel Pinto ${ }^{\mathrm{a}, \mathrm{b}}$ (jmpintog@uc.cl), Hendrik Matterne (hendrik.mattern@ovgu.de), Marcelo Andia ${ }^{\mathrm{b}, \mathrm{f}}$

(mandia@med.puc.cl), Sergio Uribe ${ }^{\mathrm{b}, \mathrm{f}}$ (suribe@med.puc.cl), and Cristian Tejos ${ }^{\mathrm{a}, \mathrm{b}}$ (ctejos@uc.cl)

a Department of Electrical Engineering, Pontificia Universidad Catolica de Chile. Avda. Vicuña Mackenna 4686, Macul, Santiago, Chile

b Biomedical Imaging Center, Pontificia Universidad Catolica de Chile. Avda. Vicuña Mackenna 4686, Macul, Santiago, Chile

c Wellcome Trust Centre for Neuroimaging, Institute of Neurology, University College London. 12 Queen Square, London, WC1N 3BG, UK

d German Center for Neurodegenerative Diseases (DZNE). Leipziger Straße 44, Haus 64, 39120 Magdeburg, Germany

e Department of Biomedical Magnetic Resonance, Institute of Experimental Physics, Otto von GuerickeUniversity. Universitaetsplatz 2,39106 , Magdeburg, Germany

f Department of Radiology, School of Medicine, Pontificia Universidad Catolica de Chile. Avda. Libertador Bernardo OHiggins 340, Santiago, Chile

Corresponding author: Carlos Milovic, cmilovic@uc.cl

Manuscript typo: Note

Abstract: Purpose: Most approaches for quantitative susceptibility mapping (QSM) are based on a forward model approximation that employs a continuous Fourier transform operator to solve a differential equation system. Such formulation, however, is prone to high-frequency aliasing. The aim of this study was to reduce such errors using an alternative dipole kernel formulation based on the discrete Fourier transform and discrete operators. Methods: The impact of such an approach on forward model calculation and susceptibility inversion was evaluated in contrast to the continuous formulation both with synthetic phantoms and in vivo MRI data.

Results: The discrete kernel demonstrated systematically better fits to analytic field solutions, and showed less over-oscillations and aliasing artifacts while preserving low- and medium-frequency responses relative to those obtained with the continuous kernel. In the context of QSM estimation, the use of the proposed discrete kernel resulted in error reduction and increased sharpness. Conclusion: This proof-of-concept study demonstrated that discretizing the dipole kernel is advantageous for QSM. The impact on small or narrow structures such as the venous vasculature might by particularly relevant 
to high-resolution QSM applications with ultra-high field MRI - a topic for future investigations. The proposed dipole kernel has a straightforward implementation to existing QSM routines.

Keywords: forward model, inverse problem, magnetic susceptibility, MRI phase, iron mapping, venography, ultra-high field MRI 


\section{Introduction}

Quantitative susceptibility mapping (QSM) has attracted considerable interest for its ability to probe local magnetostatic properties of biological tissue and for its unique dynamic range ${ }^{1}$. QSM's value chiefly lies on its sensitivity to non-heme iron, myelin and calcium content as well as venous-oxygen saturation levels and hemosiderin burden resulting from cerebral hemorrhages and microbleeds ${ }^{2}$. QSM has therefore been proposed as a useful biomarker for the study of brain aging ${ }^{3,4}$ and diseases of the central nervous system such as multiple sclerosis ${ }^{5,6,7}$, Alzheimer's disease ${ }^{8}$, Parkinson's disease ${ }^{9,10,11,12}$, amyotrophic lateral sclerosis ${ }^{13}$ and degeneration with brain iron accumulation ${ }^{14}$, where disrupted iron homeostasis, demyelination, calcification, vascular lesions and/or chronic impairment of oxygen metabolism might play relevant mechanistic roles in disease pathogenesis and progression.

Susceptibility maps can be inferred from the phase of the gradient-recalled echo (GRE) signal, which, in turn, is proportional to the local reaction field emanating from magnetic susceptibility differences between substances. Inferring susceptibility sources from phase offsets, however, is not a trivial problem. Magnetic susceptibility has long been studied from a quantum mechanics standpoint ${ }^{15}$, yet in an MRI context, source-to-field (forward) models are only a recent development, e.g. via discretization of the heat equation with finite differences or applying integral methods such as Green's function. Such approaches, however, are computationally intensive, thus they are logistically unfit for clinical purposes. The first fast-solving forward method was proposed by Salomir et al. (2003) ${ }^{16}$, who employed the Fourier transform to solve the associated differential equation. Subsequently, Marques and Bowtell (2005) ${ }^{17}$ used an integral approach applying the Fourier transform to Green's function, resulting in an analogous approximation, which also enabled a direct estimation of magnetic field offsets from an arbitrary susceptibility distribution by way of a 3D convolution operation with a magnetic dipole kernel. To date, such forward model remains to be the gold standard; although notably, such formulation is known to suffer from digitization errors due to aliasing in the vicinity of abrupt magnetic susceptibility variations ${ }^{18}$.

Early attempts to solve the field-to-source (i.e. inverse) problem for QSM faced a major limitation: the dipole kernel approximation has zero-valued coefficients across a double-conical surface in k-space, the so-called "magic cone". Therefore, direct inversion leads to divisions by zero for such frequency coefficients, which generate highly undesirable reconstruction artifacts. Truncated approximations 
improve the situation; however, such an approach also results in QSM inversions prone to streaking artifacts and noise amplification ${ }^{19}$. Consequently, field-to-source problems have been recently reformulated as an optimization problem with its functional usually comprising two terms, a data fidelity and a regularization term. The data fidelity term minimizes the error between the forward model and the MRI measurements based on a known phase noise distribution. Regularization constrains the impact of the zero-valued kernel coefficients, typically by promoting sparsity to the gradient of the computed solution. To date, a large proportion of technical QSM studies have focused on the development of novel regularization strategies, leading to substantial improvements in artifact reduction and noise management ${ }^{20}$. In the present note, however, we focused on the forward model and proposed an alternative dipole kernel formulation based on the discrete Fourier transform, aiming to reduce high-frequency aliasing in the fidelity term. We evaluated the impact of such an approach on forward model calculations and QSM inversions using both synthetic and in vivo MRI data.

\section{Material and methods}

\subsection{Theory}

It is well-known that the following differential equation can be obtained from the magnetostatic Maxwell equations ${ }^{21}$ :

$$
\nabla^{2}\left[-h_{o b j, z}\right]=H_{0} \frac{\partial^{2} X}{\partial z^{2}}
$$

where $h_{o b j, z}$ is the demagnetizing (or reaction) field due to local magnetizations, $\chi$ is the spatial distribution of susceptibility sources, $H_{0}$ is the applied (or main) magnetic field and $\nabla^{2}$ is the Laplacian operator.

Several strategies are suitable to solve Eq. (1), e.g. finite-difference, finite-element, spectral or variational methods ${ }^{22}$. Spectral methods, such as those used to derive the conventional continuous dipole kernel, would be preferred in an idealized scenario of regular geometries and smooth transitions. However, they might be less useful for studying biological tissue where strong and sharp susceptibility 
variations often occur. Alternatively, finite-element approaches are very flexible and precise, but they are computationally expensive making them impractical for QSM. As a trade-off, in the present study we propose the use of discrete Laplacian operators resulting from 3D finite-differences to define (i) the convolution kernel as a 3x3x3 cube:

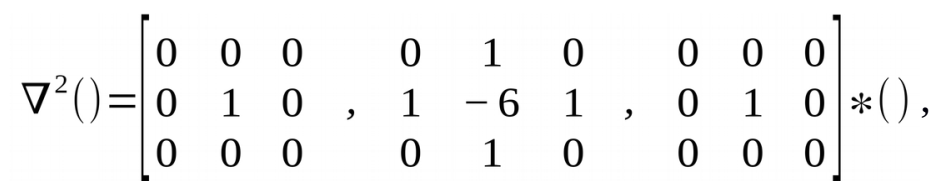

and (ii) the second derivative along the $\mathrm{z}$ axis as a $1 \mathrm{x} 1 \mathrm{x} 3$ vector, i.e.:

$$
\frac{\partial^{2}()}{\partial z^{2}}=\left[\begin{array}{llll}
1 & , & -2 & ,
\end{array}\right] *(),
$$

where * denotes a 3D convolution operation.

The new equation system can be solved using relaxation (multigrid) methods, rapid solvers or direct matrix methods. Notably, the latter-more accurate in theory— are impractical for large MRI reconstruction matrices. Relaxation methods, in contrast, are fast but they only yield approximated solutions that might be vulnerable to artifacts. Alternatively, in this study we propose the use of rapid solvers based on the discrete Fourier Transform (DFT), which are reasonably fast and more accurate than relaxation methods. Applying the DFT to Eq. (1) leads to:

$$
\begin{gathered}
-\left[-6+2 \cdot \cos \left(2 \pi \frac{k_{x}}{N_{x}}\right)+2 \cdot \cos \left(2 \pi \frac{k_{y}}{N_{y}}\right)+2 \cdot \cos \left(2 \pi \frac{k_{z}}{N_{z}}\right)\right] \cdot \operatorname{DFT}\left(h_{o b j, z}\right)=H_{0}\left[-2+2 \cdot \cos \left(2 \pi \frac{k_{z}}{N_{z}}\right)\right] \cdot \operatorname{DFT}(\chi) \\
h_{o b j, z}=-H_{0} \cdot D F T^{-1}\left(\frac{-2+2 \cdot \cos \left(2 \pi \frac{k_{z}}{N_{z}}\right)}{-6+2 \cdot \cos \left(2 \pi \frac{k_{x}}{N_{x}}\right)+2 \cdot \cos \left(2 \pi \frac{k_{y}}{N_{y}}\right)+2 \cdot \cos \left(2 \pi \frac{k_{z}}{N_{z}}\right)} \cdot D F T(x)\right), \quad \text { Eq. (2) }
\end{gathered}
$$

where $\mathrm{N}_{\mathrm{x}}, \mathrm{N}_{\mathrm{y}}$ and $\mathrm{N}_{\mathrm{z}}$ are the reconstruction matrix dimensions, and $\mathrm{k}_{\mathrm{x}}, \mathrm{k}_{\mathrm{y}}$ and $\mathrm{k}_{\mathrm{z}}$ are integer indices. 
True k-values correspond to the argument of the cosine functions.

Finally, the discrete forward model can be expressed as:

$$
\left.\Delta B_{n u c, z}=H_{0} D F T^{-1}\left\lfloor\mid \frac{1}{3}-\frac{1-\cos \left(2 \pi \frac{k_{z}}{N_{z}}\right)}{3-\cos \left(2 \pi \frac{k_{x}}{N_{x}}\right)-\cos \left(2 \pi \frac{k_{y}}{N_{y}}\right)-\cos \left(2 \pi \frac{k_{z}}{N_{z}}\right)}\right) \cdot D F T(x)\right\rfloor
$$

Let us remember the continuous solution of Eq. $(1)^{16,17}$ :

$$
\Delta B_{n u c, z}=H_{0} F T^{-1}\left[\left(\frac{1}{3}-\frac{k_{z}^{2}}{k_{x}^{2}+k_{y}^{2}+k_{z}^{2}}\right) \cdot F T(\chi)\right]
$$

Using the Taylor expansion, $1-\cos (k) \approx 1-\left(1-k^{2}+O\left(k^{4}\right)\right)=k^{2}+O\left(k^{4}\right)$, it becomes clear that overall for low frequencies the continuous and discrete kernels are analogous whereas for high frequencies, they differ. Figure 1 illustrates frequency responses for both kernels across several radial orientations, i.e. across different $\varphi$ and $\theta$ polar angles. Notably, whereas continuous kernel responses are constant across all frequencies for any given orientation, discrete kernel responses tend to be attenuated for high frequencies, in essence acting as a low-pass frequency filter. This is true for most angular representations except for a few radial profiles. Such property is the basis for preventing aliasing artifacts due to frequency truncation (Gibb's or ringing artifacts) and noise amplification in the forward model.

--- FIGURE 1 NEAR HERE ---

\subsection{Methods}

\subsubsection{Synthetic experiments}

Continuous and discrete models were compared in two synthetic experiments using the known analytic 
solution for a sphere as the ground truth ${ }^{16}$. The nuclear magnetic induction of a sphere of radius $r$, $B_{\text {nuc,sphere, }}$ can be expressed as:

$$
B_{\text {nuc }, \text { sphere }}(x, y, z)=B_{0}\left\{\left(1-\frac{2}{3} \chi_{x, y, z}\right) \cdot\left(\begin{array}{c}
\left.r^{3} \frac{x^{-} \chi_{\text {out }}}{3+\chi^{-} \chi_{\text {out }}}\left[\frac{2 z^{2}-x^{2}-y^{2}}{\left(x^{2}+y^{2}+z^{2}\right)^{5 / 2}}\right]\right), x^{2}+y^{2}+z^{2}>r^{2} \\
0, x^{2}+y^{2}+z^{2} \leq r^{2}
\end{array}\right\}\right.
$$

where $X_{x, y, z}$ is the bulk magnetic susceptibility at a given voxel coordinate, $X$ is the constant susceptibility inside the sphere, and $\chi_{\text {out }}$ is the constant susceptibility outside the sphere. For convenience, the applied field strength, $\mathrm{B}_{0}$, was set to unity.

In the first instance, a phantom with a single sphere of varying diameter was generated. Inner and background susceptibilities were set to be constant at 10 and 0 parts per million (ppm), respectively. A second synthetic phantom consisted of eight spheres of varying diameter ranging from 3 to 17 voxels, arranged in a circular pattern across the $X Y$-plane and set on a large field of view (FOV) of 256 voxels

cubed to prevent biases in the field calculation. In this experiment, all spheres also had a constant inner susceptibility of $10 \mathrm{ppm}$, with the background set to $0 \mathrm{ppm}$. The composite magnetic induction field was calculated integrating the contributions from all spheres. The complex MR signal was then calculated assuming a constant unit magnitude value across the whole FOV, except for the largest sphere, which was set to zero magnitude. Subsequently, Gaussian noise was added to the complex data (complex image domain) equivalent to a peak signal-to-noise (SNR) value of 20. Susceptibility maps were calculated from the analytic field using a modified non-linear implementation of the non-linear morphology-enabled dipole inversion (MEDI) algorithm with dynamic model error reduction (MERIT) $)^{23-26}$, incorporating both continuous and discrete kernels (original MEDI toolbox available from http://weill.cornell.edu/mri/pages/qsm.html). Regularization weights were optimized using the Lcurve approach $^{27}$. Other MEDI parameters were set to default values.

\subsubsection{In vivo human experiment}

A healthy subject (27 year-old female) was scanned (written consent was obtained in accordance with the Declaration of Helsinki) on a 7 Tesla whole body MRI system (Siemens Healthcare, Erlangen, 
Germany) using a quadrature transmit, 32-channel reception head coil (Nova Medical Inc., Wilmington, Massachusetts, USA). A 3D, RF-spoiled, fully-flow compensated GRE sequence was used with an optical prospective motion correction system - correcting motion for each k-space line ${ }^{28}$, and the following imaging parameters: repetition time $/$ echo time $=20 / 9 \mathrm{~ms}$; flip angle $=10^{\circ}$; bandwidth $=$ $120 \mathrm{~Hz} /$ pixel; matrix dimensions 504×608×88 and voxel size $0.33 \times 0.33 \times 1.25 \mathrm{~mm}^{3}$. The full k-space acquisition gave a scan time of 17:30 minutes.

Complex channel data were processed as follows: (i) brain mask calculation (from root sum-of-squares modulus combination) using BET2 ${ }^{29}$ with a lenient fractional threshold of 0.1 ; (ii) channel-wise phase unwrapping and harmonic phase removal using HARPERELLA ${ }^{30}$ (STI Suite v1.42 http://people.duke.edu/ cl160), with 100×100×50 zero-padding and 200 iterations; (iii) magnitudeweighted, HARPERELLA-normalized channel phase combination; (iv) local field estimation using the spherical mean value filtering method with varying spherical kernels (VSHARP without deconvolution, also available from the STI Suite) ranging from $25 \mathrm{~mm}$ radius at the center of the brain to $1 \mathrm{~mm}$ at the

brain boundary ${ }^{31}$; finally, (v) susceptibility inversions using continuous and discrete implementations of the MEDI algorithm (500 iterations for the internal conjugate gradient loop and 0.05 normalized residual as stopping criterion). In vivo GRE data were reconstructed for a large range of regularization parameters to generate L-curves. Subsequently, a blind rater (J.A.-C.) was presented with a randomized subset of QSM inversions ( $\mathrm{n}=6$ continuous, $\mathrm{n}=6$ discrete) including L-curve based optimal reconstructions, which were visually rated from 1 to 5 (1-2: under-regularized; 3: optimal; 4-5: overregularized) for contrast with the L-curve optimization approach.

\section{Results}

\subsection{Forward model estimation using single-sphere phantoms}

The experiment illustrated in Fig. 2 revealed that model accuracy is sphere-caliber dependent. Errors were observed at sphere/background interfaces, most remarkably for smaller spheres (Fig. 2a and 2c). For large spheres, discrepancies close to FOV boundaries between analytic and both numerical models were greater than those at interfaces (Fig. 2b), presumably due to periodic boundary errors generated by the Fourier transform. A closer inspection in the vicinity of sphere interfaces (Fig. 2c and 2d) 
revealed smoother solutions with fewer over-oscillations due to aliasing for the discrete kernel, i.e. more accurate results overall than those for the continuous kernel. Fig. 2e illustrated that for sphere diameters of up to $10 \%$ (relative to the total FOV, i.e. 25 pixels), error reduction for the discrete kernel was at least $5 \%$.

--- FIGURE 2 NEAR HERE ---

\subsection{QSM inversion for the eight-sphere phantom}

QSM estimates were systematically more accurate for all sphere calibers using the discrete kernel (Fig. 3a). A closer inspection of the resulting QSM inversions inside the simulated spheres revealed stronger Gibbs ringing for the continuous kernel (Fig. 3b and 3d) than for the proposed discrete formulation (i.e. less structured artifacts in Fig. 3c and 3e).

--- FIGURE 3 NEAR HERE ---

\subsection{QSM inversion for in vivo human data}

Both human (blind rater) and automated (L-curve based) approaches agreed on the optimal QSM reconstruction both for continuous and discrete kernels (Fig. 4). The optimal QSM reconstruction for the continuous kernel is shown in Fig. 5a. The difference map between both (continuous minus discrete) optimal reconstructions (Fig. 5b) highlighted remnant streaking and checkerboard errors (Fig. 5c), presumably due to the discrete kernel ability to suppress such errors. In an attempt to identify the origin of such differences (due to the lack of a ground truth), two additional difference maps were computed, continuous (5c) and discrete (5d), relative to an over-regularized (continuous) solution. Over-regularization attenuates the strength of streaking, yet at the expense of edge definition at the interface between tissues with different magnetic susceptibility. The standard deviation of the difference between the optimal continuous result and the over-regularized solution was approximately 2\% larger (4.54 ppb) than that for the discrete kernel (4.46 ppb). Such differential behavior can be explained by larger oscillations in the medium-to-large scale components, which substantiate the notion that the optimal continuous solution includes more streaking artifacts and errors in the neighborhood of strong susceptibility variations. 
--- FIGURE 4 NEAR HERE ---

--- FIGURE 5 NEAR HERE ---

\section{Discussion}

It has been suggested the current state-of-the-art continuous forward model inferred by Salomir et al., $2003^{16}$ and Marques and Bowtell, $2005^{17}$ is prone to aliasing/digitization errors due to limited bandwidth in the MRI measurement ${ }^{18}$. To address the shortcoming, we propose the use of discrete Laplacian operators resulting from 3D finite-differences and the discrete Fourier transform to derive the dipole kernel. The proposed discrete forward model showed an improvement over the continuous counterpart, i.e. it generated smoother results and returned more accurate field representations at sharp magnetic susceptibility interfaces (Fig. 2c and 2d). Such behavior was markedly noticeable for small synthetic spheres (Fig. 2e), whereas large-scale responses were similar for both kernels (Fig. 2b) with discretization-independent errors plausibly driven by the intrinsic limitations of the Fourier transform and its periodic boundary conditions. In theory, finite-difference operators could introduce errors; however, in practice, this study revealed improved performance for the proposed discrete formulation near sharp susceptibility boundaries where the continuity assumptions of spectral methods are often violated (Fig. 2). An important consequence of the proposed discretization scheme is rotational asymmetry around the z-axis for high-frequency kernel responses (Fig. 1). Although undesirable on theoretical grounds, this is often irrelevant since most susceptibility weighted MRI prescriptions are prescribed for resolution efficiency with anisotropic voxels $s^{1}$, which readily impose rotational asymmetries in the acquired data.

A major motivation for refining the forward model was to improve QSM inversion. We therefore evaluated the performance of the proposed discrete dipole kernel for susceptibility estimation using a 3D volume containing eight spheres of different caliber. Such an experiment also yielded discrete kernel solutions that were consistently more accurate, independently of sphere size, as a result of the successful suppression of over-oscillations at sphere boundaries (Fig. 3). Reduced Gibbs ringing was an expected result since the proposed discrete kernel partly behaves as a low-pass frequency filter with a high cut-off frequency (see Fig. 1). For the inverse problem, consequently, the new kernel acts as a deconvolution filter. Such property might have an impact on the regularization term, as it might avoid 
excessive attenuation of true local variances often resulting from inaccurate model error compensation. This would also imply that Lagrange multipliers (i.e. regularization parameters) might not necessarily represent the same consistency/smoothing interplay for both kernel types, leading to differential Lcurve relationships and ultimately, different optimal parameters. The latter was confirmed with in vivo MRI data using L-curve (point of maximum curvature) and visual rating methods (Fig. 4). Most notably, the optimal discrete kernel solution yielded better suppression of streaking and checkerboard artifacts (than the optimal continuous kernel QSM) without apparent loss of anatomical detail (Fig. 5). The sharpening properties of the discrete kernel in the inverse problem scheme enable the use of stronger regularization weights, which in turn helped reduce noise and streaking artifacts without loss of anatomical detail.

There are certain applications, for example QSM-based venography or the study of small iron-rich nuclei, where the sharpening properties of the newly proposed kernel might result in relevant improvements to the measurement. Although this was not addressed specifically in this study, a future investigation is warranted. As a final note, the re-formulation of the dipole kernel is a straightforward implementation to existing QSM routines, which results in highly comparable processing times.

\section{Conclusions}

We presented a new formalism for the forward problem of estimating a local induction field from an arbitrary magnetic susceptibility distribution. The proposed approach uses finite-difference operators and the discrete Fourier transform to solve the associated differential equations. Such an approach yielded an alternative dipole kernel that demonstrated better fits to analytic solutions, and showed less over-oscillations and aliasing artifacts while preserving low- and medium-frequency responses relative to the continuous kernel. When applied to the inverse problem, the proposed kernel generated less errors and streaking artifacts, suggesting QSM with kernel discretization is preferable for the study of small deep brain nuclei or the subtleties of the venous vasculature with ultra-high field MRI - a topic for future research.

This study opens the door to a new area of QSM research, where additional improvements to the forward model might also result from using finite elements, or from replacing the Fourier transform 
entirely by other methods to solve the associated differential equations. In addition, establishing more appropriate boundary conditions might also contribute to improving forward model estimates at large and medium scales which, in turn, may enable the prescription of reduced FOV acquisitions, or may circumvent the need for zero padding.

\section{Acknowledgments}

We thank FONDECYT 1161448, CONICYT Programa PIA Anillo ACT1416, Becas de Doctorado Nacional CONICYT Folio 21150369, and the National Institutes of Health, grant number 1R01DA021146, for their funding support. We also thank Alessandro Sciarra for assisting with the in vivo measurement and Prof Oliver Speck for logistical support.

\section{References}

1. Haacke EM, Liu S, Buch S, Zheng W, Wu D, Ye Y. Quantitative susceptibility mapping: Current status and future directions. Magn Reson Imaging. 2015;33:1-25.

2. Reichenbach JR, Schweser F, Serres B, Deistung A. Quantitative Susceptibility Mapping: Concepts and Applications. Clin Neuroradiol. 2015;25:225-230.

3. Acosta-Cabronero J, Betts MJ, Cardenas-Blanco A, Yang S, Nestor PJ. In Vivo MRI Mapping of Brain Iron Deposition across the Adult Lifespan. J Neurosci . 2016;36:364-374.

4. Bilgic B, Pfefferbaum A, Rohlfing T, Sullivan EV, Adalsteinsson E. MRI estimates of brain iron concentration in normal aging using quantitative susceptibility mapping, NeuroImage. 2012;59:26252635.5. Langkammer C, Liu T, Khalil M, Enzinger C, Jehna M, Fuchs S, Fazekas F, Wang Y, Ropele S. Quantitative susceptibility mapping in multiple sclerosis. Radiology. 2013;267:551-559.

6. Wisnieff C., Ramanan S., Olesik J., Gauthier S., Wang Y. Pitt D. Quantitative susceptibility mapping (QSM) of white matter multiple sclerosis lesions: Interpreting positive susceptibility and the presence of iron. Magn. Reson. Med. 2015;74:564-570.

7. Chen W, Gauthier SA, Gupta A, Comunale J, Liu T, Wang S, Pei M, Pitt D, Wang Y. Quantitative susceptibility mapping of multiple sclerosis lesions at various ages. Radiology 2014;271(1):183-192.

8. Acosta-Cabronero J, Williams GB, Cardenas-Blanco A, Arnold RJ, Lupson V, Nestor PJ. In vivo quantitative susceptibility mapping (QSM) in Alzheimer's disease. PLoS ONE. 2013;8:e81093.

9. Guan X, Xuan M, Gu Q, Huang P, Liu C, Wang N, Xu X, Luo W, Zhang M. Regionally progressive accumulation of iron in Parkinson's disease as measured by quantitative susceptibility mapping. NMR Biomed. 2016;doi: 10.1002/nbm.3489. 
10. Du G, Liu T, Lewis MM, Kong L, Wang Y, Connor J, Mailman RB, Huang X. Quantitative susceptibility mapping of the midbrain in Parkinson's disease. Mov Disord. 2016;31:317-324

11. Azuma M, Hirai T, Yamada K, Yamashita S, Ando Y, Tateishi M, Iryo Y, Yoneda T, Kitajima M, Wang Y, Yamashita Y. Lateral Asymmetry and Spatial Difference of Iron Deposition in the Substantia Nigra of Patients with Parkinson Disease Measured with Quantitative Susceptibility Mapping. AJNR Am J Neuroradiol. 2016;37:782-788

12. Acosta-Cabronero J, Cardenas-Blanco A, Betts MJ, Butryn M, Valdes-Herrera JP, Galazky I, Nestor PJ, The whole-brain pattern of magnetic susceptibility perturbations in Parkinson's disease. Brain. 2017 1;140:118-131

13. Schweitzer AD, Liu T, Gupta A, Zheng K, Seedial S, Shtilbans A, Shahbazi M, Lange D, Wang Y, Tsiouris AJ. Quantitative Susceptibility Mapping of the Motor Cortex in Amyotrophic Lateral Sclerosis and Primary Lateral Sclerosis. AJR Am J Roentgenol. 2015;204:1086-1092.

14. Löbel U, Schweser F, Nickel M, Deistung A, Grosse R, Hagel C, Fiehler J, Schulz A, Hartig M, Reichenbach JR, et al. Brain iron quantification by MRI in mitochondrial membrane protein-associated neurodegeneration under iron-chelating therapy. Ann Clin Transl Neurol. 2014;1:1041-1046.

15. White RW. Quantum theory of magnetism. Berlin: Springer-Verlag; 2nd Ed, 1982. p 186-193

16. Salomir R, de Senneville BD, Moonen CT. A fast calculation method for magnetic field inhomogeneity due to an arbitrary distribution of bulk susceptibility. Concepts Magn Reson Part B. 2003;19B:26-34.

17. Marques JP, Bowtell R. Application of a Fourier-based method for rapid calculation of field inhomogeneity due to spatial variation of magnetic susceptibility. Concepts Magn Reson Part B: Magnetic Resonance Engineering. 2005;25B:65-78.

18. Cheng Y-CN, Neelavalli J, Haacke EM. Limitations of calculating field distributions and magnetic susceptibilities in MRI using a Fourier based method. Phys Med Biol. 2009;54:1169-1189.

19. Shmueli K, de Zwart J a, van Gelderen P, Li T-Q, Dodd SJ, Duyn JH. Magnetic susceptibility mapping of brain tissue in vivo using MRI phase data. Magn Reson Med. 2009;62:1510-1522.

20. Wang S, Liu T, Chen W, Spincemaille P. Noise effects in various quantitative susceptibility mapping methods. IEEE Trans Biomed Eng. 2013;60:3441-3448.

21. de Rochefort L, Nguyen T, Brown R, Spincemaille P, Choi G, Weinsaft J, Prince MR, Wang Y. In vivo quantification of contrast agent concentration using the induced magnetic field for time-resolved arterial input function measurement with MRI. Med Phys. 2008;35:5328-39.

22. Press WH, Teukolsky SA, Vetterling Wt, Flannery BP. Numerical Recipes in C: the art of scientific computing, Second Edition. Cambridge Univ. Press, New York. 1992.

23. Liu T, Wisnieff C, Lou M, Chen W, Spincemaille P, Wang Y. Nonlinear formulation of the magnetic field to source relationship for robust quantitative susceptibility mapping. Magn Reson Med. 
2013;69:467-476.

24. Liu J, Liu T, De Rochefort L, Ledoux J, Khalidov I, Chen W, Tsiouris AJ, Wisnieff C, Spincemaille $\mathrm{P}$, Prince $\mathrm{MR}$, et al. Morphology enabled dipole inversion for quantitative susceptibility mapping using structural consistency between the magnitude image and the susceptibility map. NeuroImage. 2012;59:2560-2568.

25. Liu T, Liu J, de Rochefort L, Spincemaille P, Khalidov I, Ledoux JR, Wang Y. Morphology enabled dipole inversion (MEDI) from a single-angle acquisition: comparison with COSMOS in human brain imaging. Magn Reson Med. 2011;66:777-783.

26. de Rochefort L, Liu T, Kressler B, Liu J, Spincemaille P, Lebon V, Wu J, Wang Y. Quantitative susceptibility map reconstruction from MR phase data using bayesian regularization: validation and application to brain imaging. Magn Reson Med. 2010;63:194-206.

27. Hansen PC. The L-Curve and its Use in the Numerical Treatment of Inverse Problems. in Computational Inverse Problems in Electrocardiology, ed. P. Johnston, Advances in Computational Bioengineering. 2000;4:119-142.

28. Maclaren J, Armstrong BSR, Barrows RT, Danishad KA, Ernst T, Foster CL, Gumus K, Herbst M, Kadashevich IY, Kusik TP, et al. Measurement and Correction of Microscopic Head Motion during Magnetic Resonance Imaging of the Brain. PLoS ONE. 2012;7:3-11.

29. Smith SM. Fast robust automated brain extraction. Hum Brain Mapp. 2002;17:143-155.

30. Li W, Avram A V, Wu B, Xiao X, Liu C. Integrated Laplacian-based phase unwrapping and background phase removal for quantitative susceptibility mapping. NMR Biomed. 2014;27:219-227.

31. Li W, Wu B, Liu C. Quantitative susceptibility mapping of human brain reflects spatial variation in tissue composition. NeuroImage. 2011;55:1645-1656. 


\section{Figure captions:}
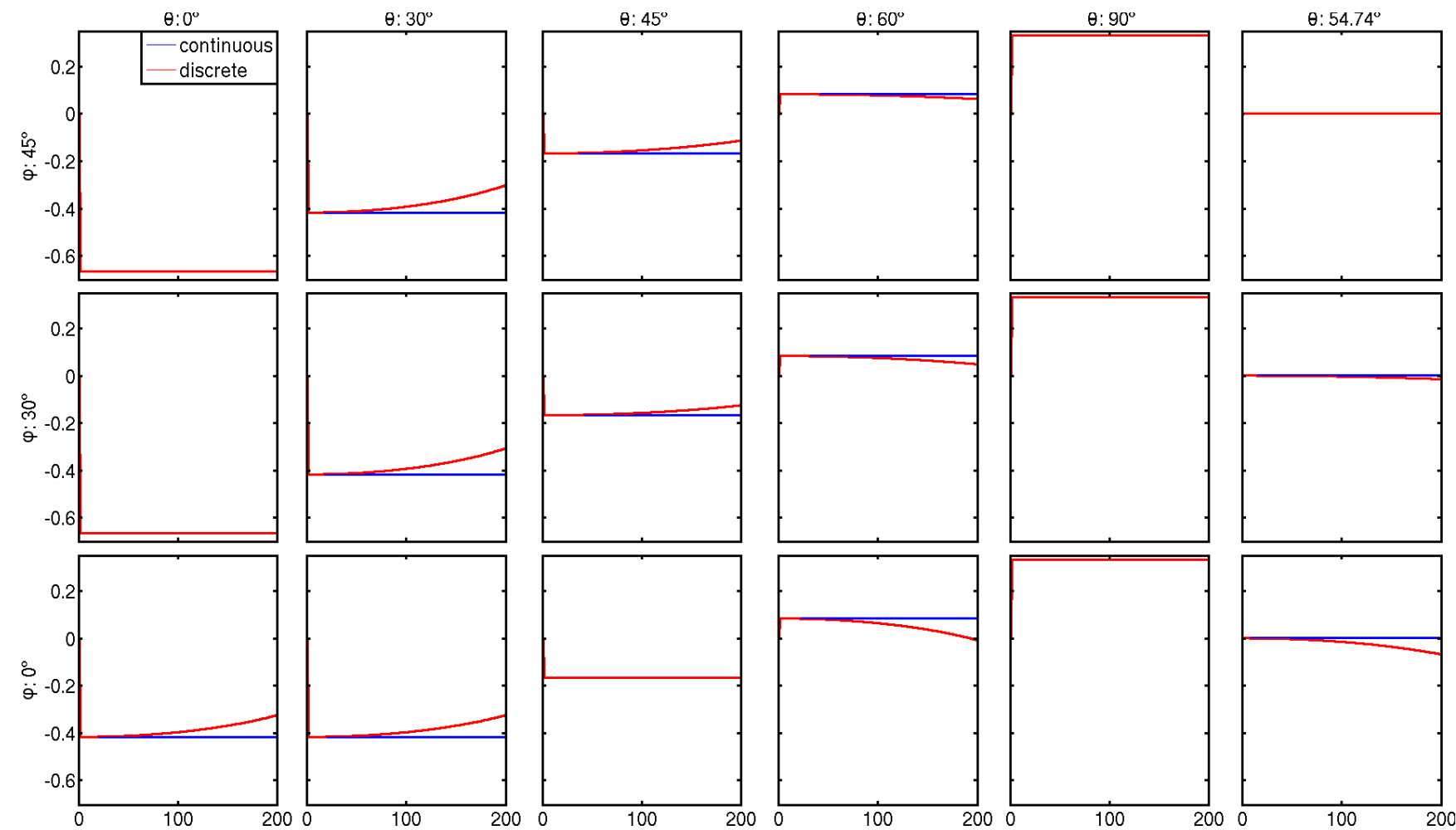

Figure 1. Frequency responses ( $Y$-axes) for continuous (blue) and discrete (red) dipole kernels. $X$-axes represent integer indices around the k-space center (for a volume of 401 voxels, each side). Only positive indices are displayed. Each panel row represents a different $\varphi$ angle; columns denote $\theta$ angles, in the spherical coordinate system (ISO convention). 

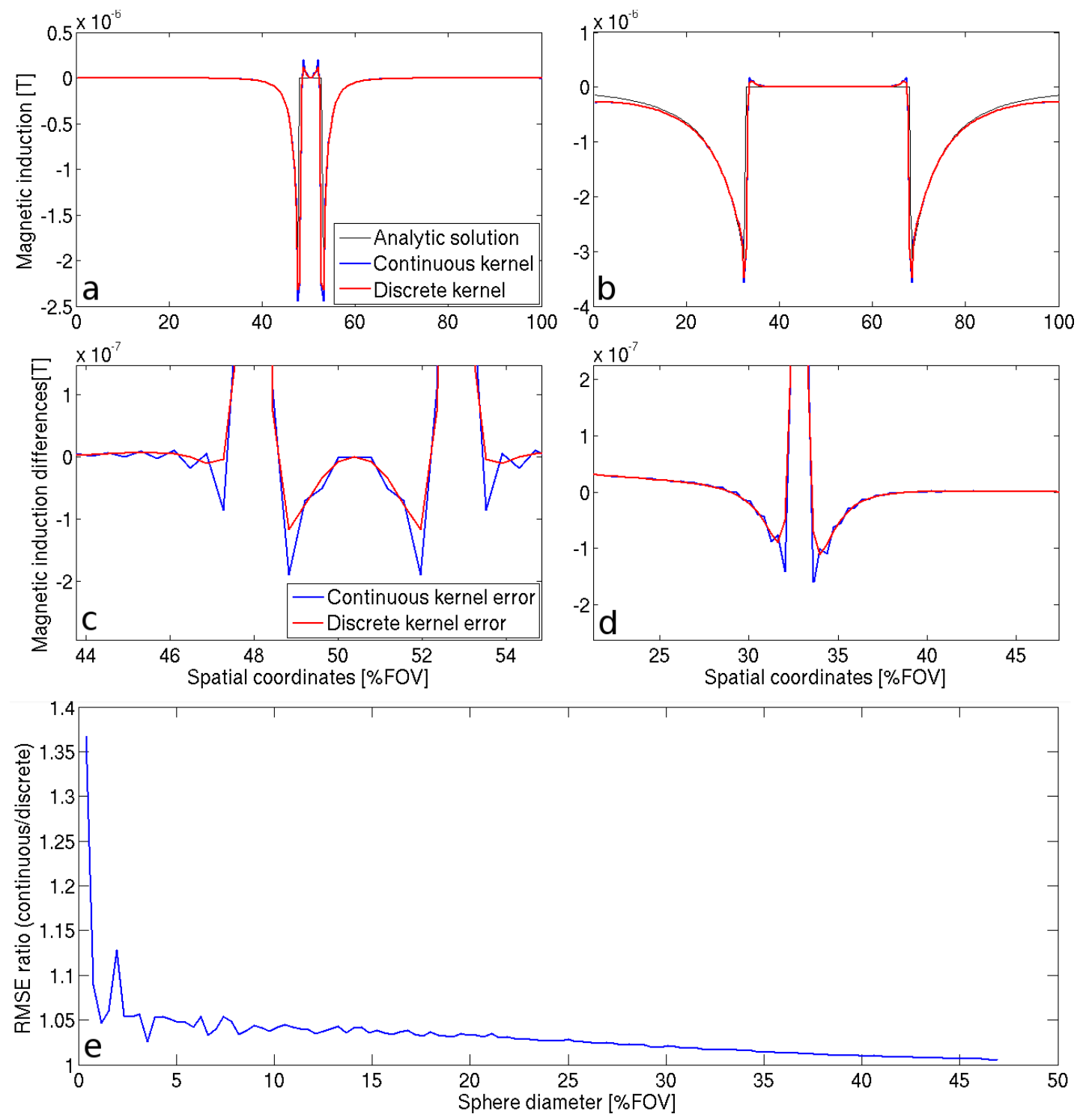

Figure 2. Magnetic inductions across the center of the Z-axis calculated with numerical and analytic solutions for (a) 9-voxel and (b) 51-voxel diameter spheres. Magnified view of the sphere/background interface for the difference between analytic and both numerical models in (c) 9-voxel and (d) 51-voxel diameter spherical phantoms. (e) Mean-root-square error (MRSE) ratio for continuous relative to discrete numerical solutions of the forward model as a function of sphere caliber. 


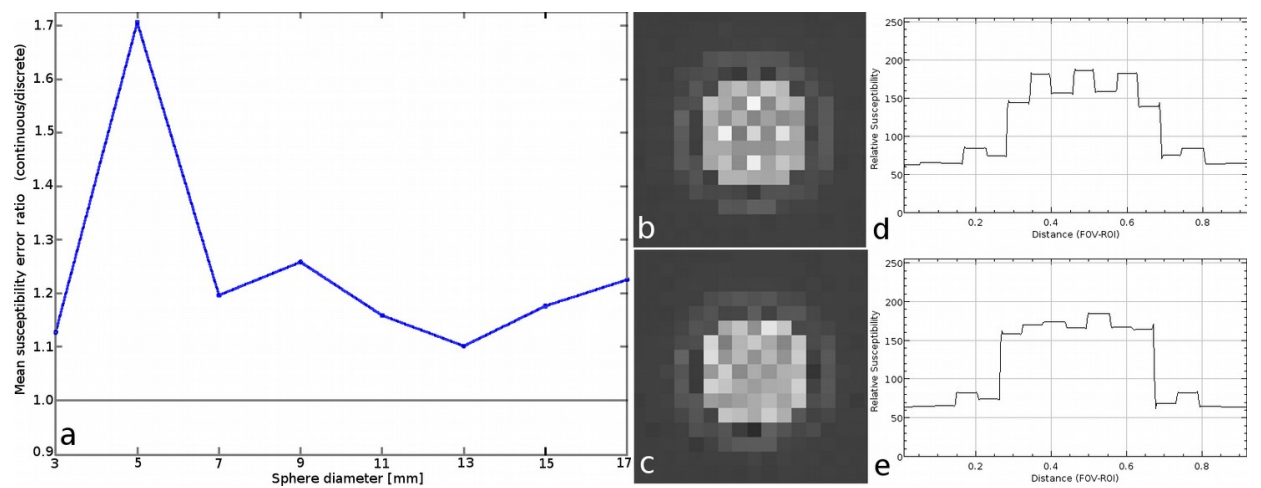

Figure 3. Phantom reconstructions. (a) Mean susceptibility error ratio for continuous relative to discrete numerical solutions as a function of sphere caliber. QSM reconstruction magnified view of the 7-pixel diameter sphere using (b) continuous and (c) discrete kernels, along with profile plots for the mean of three lines in the coronal axis, same spheres, for the (d) continuous and (e) discrete kernels.

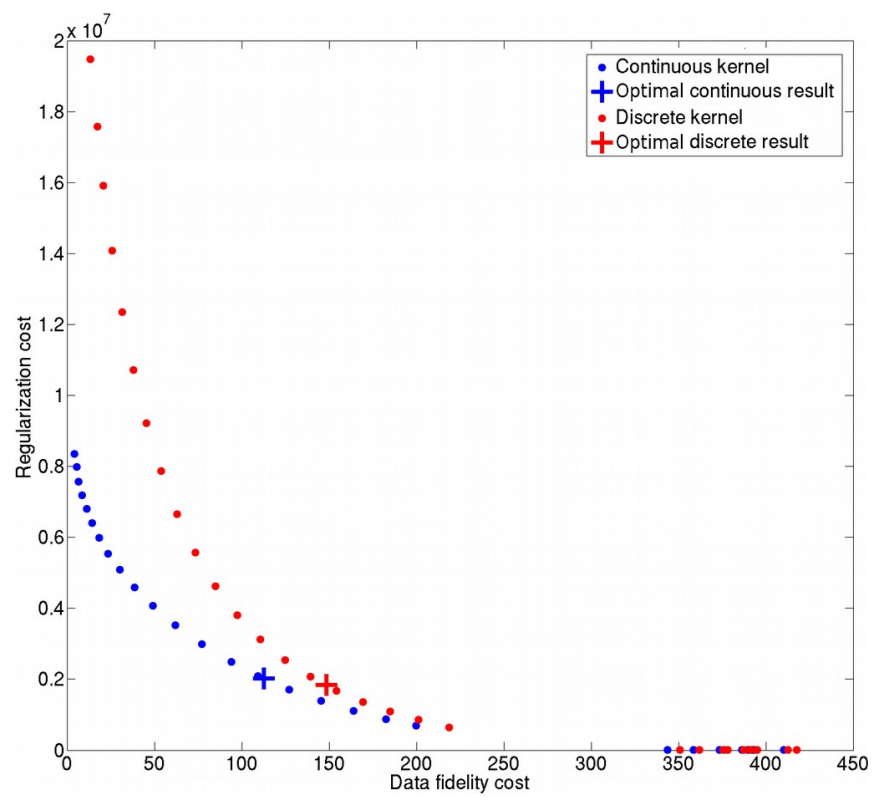

Figure 4. L-curves for in vivo MRI data using continuous (blue) and discrete (red) kernels. 

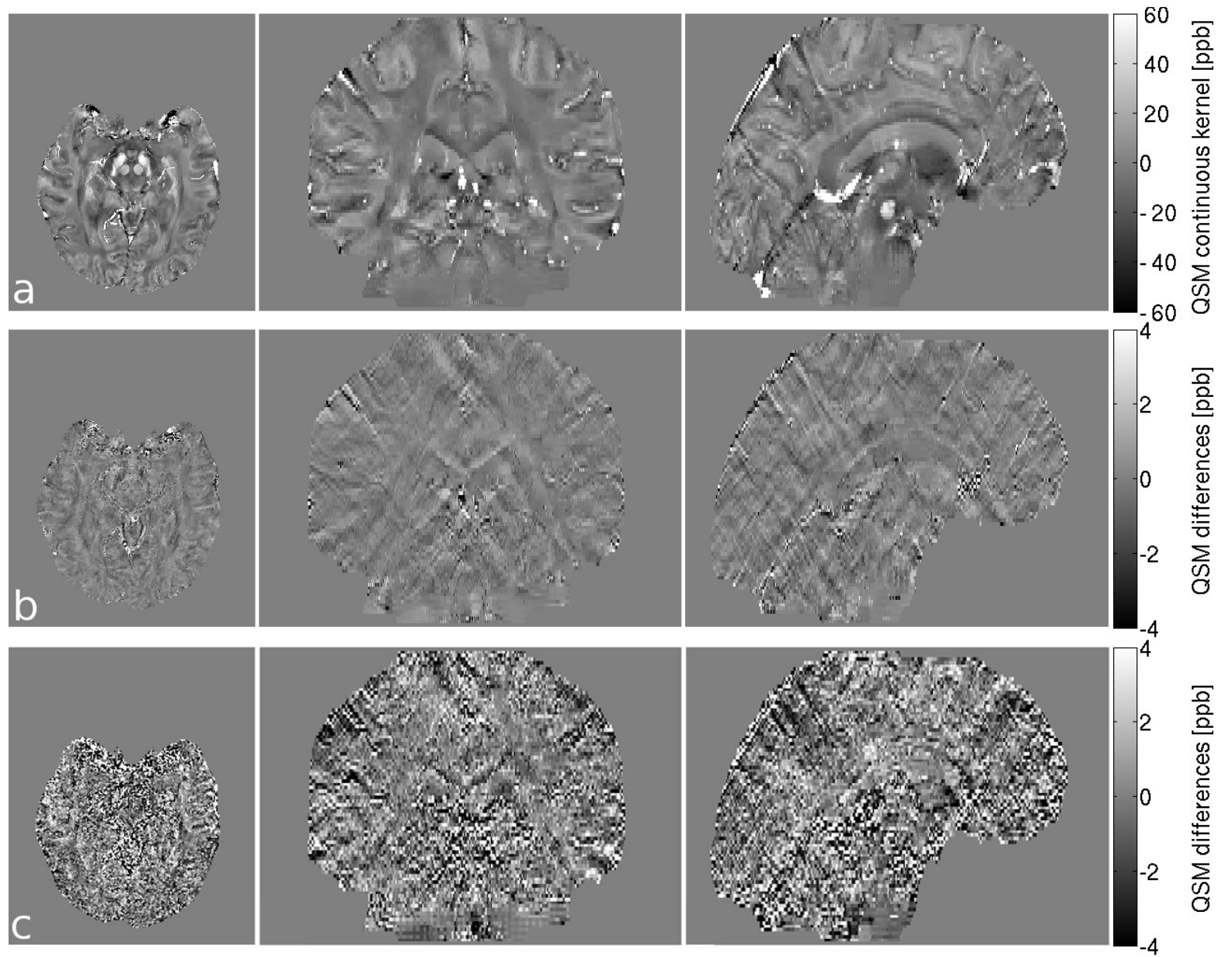

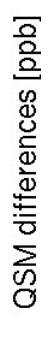
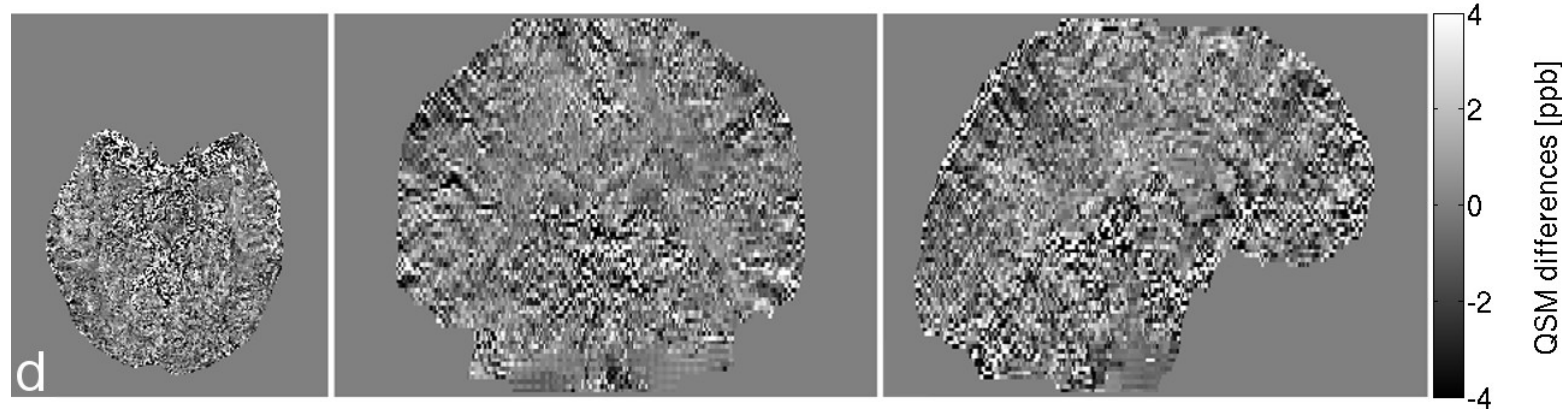

Figure 5. In vivo QSM reconstructions. (a) Axial, coronal and sagittal cuts for optimal QSM using the continuous kernel. (b) Difference between continuous and discrete kernel solutions. (c) Difference between optimal continuous solution and an over-regularized continuous solution (lambda = 150). (d) Difference between optimal discrete solution and the same over-regularized continuous solution. 